\title{
ANALYSIS OF APPROACHES FOR FILTERING MESSAGES ON SO- CIAL NETWORKING SITES
}

\author{
${ }^{1}$ HARSHALI Y. DEORE, ${ }^{2}$ S S. HATKAR \\ ${ }^{1}$ M. Tech. Student, SGGS IE\&T, Nanded-431606, India. \\ ${ }^{2}$ Assistant Professor, SGGS IE\&T, Nanded-431606, India. \\ E-mail: ${ }^{1}$ harshali.deore89@gmail.com, ${ }^{2}$ sshatkar@sggs.ac.in
}

\begin{abstract}
With the increasing use of social networking sites privacy of the user become an utmost important issue .The social networking sites do provide some security for users where they can control and filter display of messages i.e. who can display messages on their confidential area which is generally called wall. This paper analyses mainly social networking sites such as Facebook ,twitter etc. Though these sites provide some extent of security the issue of what kind of messages will be displayed on the wall of a user is still remain. This Paper covers the approaches of content based filtering i.e. filtering of messages based on the text. OSN user walls is one of the approach covered. It provides classification mechanism for users to avert the data that is not meaningful for user. OSN thus enables users to self-regulate the messages posted on their area and to control the area whether private or public. The two important techniques to be used are rule-based system and a Machine Learning classification which is used to decide filtering criteria on walls and data filtering.
\end{abstract}

Keywords-Machine Learning, Rule-Based, OSN.

\section{INTRODUCTION}

On online social networks (OSN) there is huge exchange of information each day. The information that is being shared is in different forms like audio, video, image and text. From this huge information particular user can be provided with data of its interest by using filtering techniques. This is accomplished using many methods like OSN user walls which emphasizes on filtering based on content or data. As in the exchange of information the major share is of text as many messages contain few words text where the restriction come for the existing algorithms as there is infrequent occurrence of words.

The aim of the OSN user walls is hence filtering of messages by using Filtered Wall which is used to filter undesirable messages from OSN user walls. Using machine learning categorization techniques [2].A set of category will be created with each text of few words where category will be created based on the data message is holding.

The powerful idea in OSN user walls is use of filtering rules (FR) using them user can decide which messages or content should be allowed to show on their walls. There are many things based on which filtering rules can be made such as type of data user shares, contacts of user In his list as well as result of machine learning categorization to decide the filtering criteria. Alongwith its own filtering criteria for data the system also supports the security measure provided by online social network sites like blocking of the other users to control the messages posted by them and messages which should not be displayed to them. Text Categorization Shell is another approach for categorization of information. With TCS it is possible to categorize the text and grouping it into categories which can be used to know the interest of users and providing the mesages to them based on this. TCS comes up with any requirement for automatic text categorization such as preciseness, alacrity, flexibility with other applications and less efforts to maintain and develop it. The system which are in use for automatic text categorization falls under two categories which are information retrieval techniques and knowledge based techniques [3].Consecutive sections of this paper include detail explanation of the approaches and methods used for classification of data and filtration of content.

\section{RELATED WORK}

Relate work includes:
A. Latent Semantic Indexing
B. Categorization of short text
C. Rules for filtering and Blacklist execution
D. Suggested architecture for Filtering

\section{A. LATENT SEMANTIC INDEXING}

In latent index semantics, correlation between the phrase and the document is calculated which works in information retrieval. In each document the use of a phrase or a word follows some pattern which is used to predict the structure which is then used to retrieve the information. LSI analysis uses single value decomposition (SVD) which uses eigenvector method for putrefaction. SVD uses method of relating words with documents which are composed into matrix and then the matrix is splits into set of 80 to 280 elements from which we can get the native matrix by linear combination. The documents are not represented as 
vectors of words which does not relate to each other LSI represents them as contiguous values on each of the $\mathrm{j}$ dimensions which are obtained from SVD. Eachword will not have unique vector to be assigned as 2 or more words can use same vector in minimized dimension of LSI. The relation between documents and words is represented by their location in the vectors. The similarity is decided by taking the product of vectors. Vectors in space are identified by using phrase in a query and accordingly the documents rank is decided.

\section{Information Retrieval Technique for filtering}

Latent semantic index and keyword matching technique both follow making of vector matrix where vectors represent word in document or documents by words. This pattern decides the values in a matrix or space of measures. Those documents which share almost similar content or which maximum words in common they possess the nearby values that is they share the indexes which are near to each other and have less difference. This distinctive attribute is used as base for filtration. When any new documents comes in, it is compared with the documents in a space and if there is any kind of match in both of these documents then it is considered as of same interest. If no match is found considered as document of different topic or interest and it occupies a new position in a space.

Latent semantic index method and keyword matching both are almost similar with the only difference that in keyword matching words are directly compared within the available pool of documents and in latent semantic index the index value is decided for each document and depending on that value it is placed in vector space or matrix. There can be different kind of articles for filtering like articles of daily news or articles which are related to technical topics. Those articles which are related to technical topics do not differ in a small period of time so it becomes easy to compare them as the words remain same over the time period. But in newsfeed kind of articles users interest might vary from day to day basis so deciding one particular pattern of interest in such kind of articles is quite difficult. From the experiment [8] it is proven that latent semantic index improves the performance over keyword matching by an average of $20 \%$ and also the advancement over precision.

\section{B. CATEGORIZATION OF SHORT TEXT}

The machine learning aspect is used in this technique where instead of entirely filtering the messages and deciding whether they are of interest or not, the procedure is classified into two steps where initial step is finding the sentences which are not related at all and removing them. In second step, the sentences which are similar somewhat with subject of interest observed. In this method first the words are simply classified into "Hard" and "Soft" categories. In first step hard words are simply removed and in second step instead of simply deciding which words are related to the topic, gradually they are observed and classified into different sets where value or index for them is decided. Later this data is used for further processing in filtration method.

\section{Representation of a text}

Though diversified features are proposed yet for categorization of short text the most relevant feature representation of short text messages is yet to invented. There are three types of features which are, BoW, document properties and contextual features (CF). The initial two types of features are viscerous, that is they totally rely on the data of the message that is content of the message. These kind of features derives good results but in functional environment it is appropriate to use the extrinsic information that is information from any origin that is somehow related to the text in message. To symbolize the ambience where user is posting there is proposal of CF modelling data. These all element play an important role in knowing the linguistic of the messages. From all the existing and suggested features the combination of all these are used to classify the short message or short text.

The model for representation of text is Vector Space Model (VSM) conferring with a document $\mathrm{dk}$ which contains the text is indicated as vector of binary or real weights where $d k=w t 1, \ldots \ldots \ldots \ldots \ldots, w t T k$ where the number of features are represented by $\mathrm{T}$ which comes across at least once in the pool of documents. Benefaction of the feature for the document is also measured. The standard formula is defined for computing the weight of feature in document $\mathrm{dk}$ that is how many times the term occurs in the document. The parameters which this combination of methods uses for filtration are filtration of related words, unrelated words, upper case words, and the other special characters.

\section{RULES FOR FILTERING AND BLACKLIST EXECUTION}

Social networking sites represent the same structure like graph where nodes represent the users and edges between the nodes represent relation between the users. This structure decides the faith level of an user in his network. So there are different rules for direct and indirect relations between the users. Here we take into account 3 important issues that can affect on the filtration process. First is in online social networks where huge information is shared everyday there can be repetition of same type of information. So we can apply the filtration criteria on the user who creates the messages.

The criteria can be decided from users social graph and his choices of selecting data or content online. Blacklist rules are different from rules of filtering. Here user can block the person even if person is not in direct relation thus managing him from posting any 
kind of stuff on his wall. BL rules also allow users to identify user profiles and according to them blocking them using their graph on social site. The user can also provide some time period for this blocking. He can block the person for some specific time or can block permanently. There is some threshold which is created based on the number of times the user is putted into blocking list. Depending on the threshold if the user crosses that beyond point he then considered to be liable to put into the blocking list as threshold indicates his bad behavior.

This criteria can be applied only to those who have been inserted into the blacklist at least once. If the users messages continue to fail the filtering rules we can map it using the relative frequency. There has to be some rules $\mathrm{t}$ be followed for performing $\mathrm{BL}$ mechanism. Some of the rules are: 1) how the mechanism first will detect the users who are need to be banned 2) For how much duration they have to be banned The rules defined does not apply for general high level that is covering all users under same rule. These rules are user specific. The user who is blocked for wall or personal are of one user can be able to post on some other user's wall.

\section{SUGGESTED ARCHITECTURE FOR FITERING}

This architecture focused on filtering of messages based on information or data contain in the message. Specifically this architecture focused on providing language independent filtering of messages. It follows the same approach of graph that is nodes and edges. This architecture is of 3 levels. The first layer manages profiles of users and their relationships. In addition to this some of the social networking sites provide support for other Social Networking Applications. Sometimes this additional layer may require support of Graphical User Interface. The system which is suggested here can be placed into second or third layer as it is thought as social networking application. Specifically users communicate with the system by means of GUI and decides their filtering criteria according to which the information or data can be filtered. Graphical user interface supports firewall where information or data is filtered according to rules set that is according to the criteria mentioned by the user.

These are considered as authorized messages. The important elements of this architecture are data or information based filtering and short text categorization of the messages. These are the two important modules. There are different categories according to which short text categorization module works and after filtration puts the message into one of the categories. Once the category is given by the second module first module imposes the categorization rules according to the filtering criteria. For this system takes help of blacklist management according to which the messages from the undesired users can be prevented. Time for blocking of the users can also be specified. Short text classifier gives an excellent result when it works on pool of documents. Short messages can also be classified using the approach of neural and neural classification technique[5].

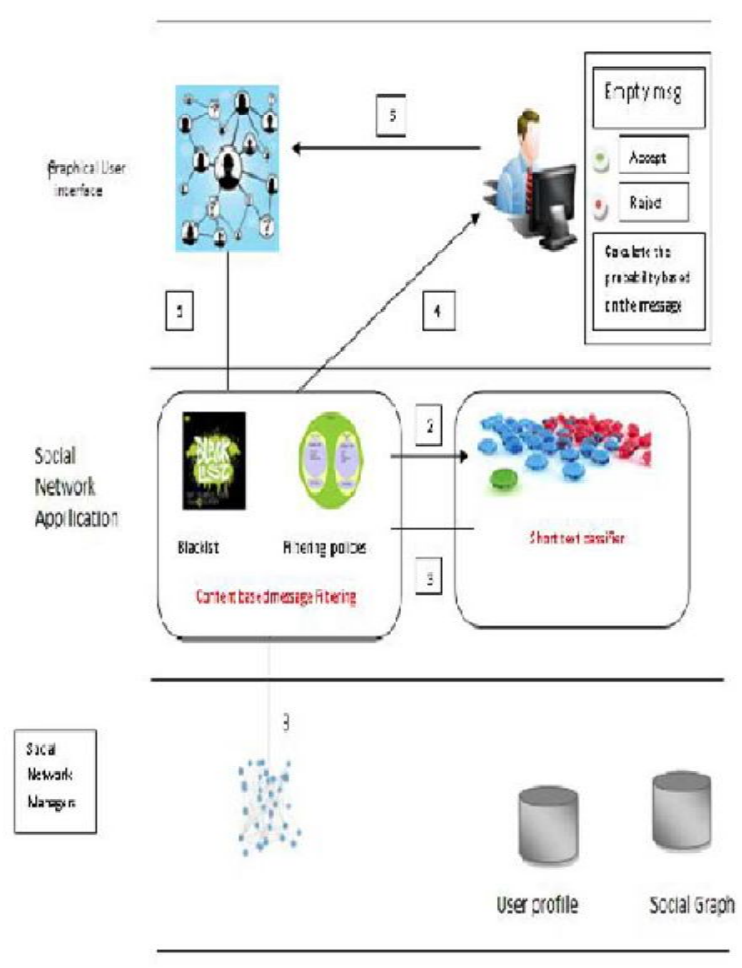

Figure 1Architecture of Filtration

\section{LITERATURE SURVEY}

\section{Relationship guideline for data based filtering}

The suggestions which explore categorization techniques for acquiring online social network categorization method in order to astounding users from loads of data which is not precise. With this the user is able to see the posts or tweets of his area of interest only. There are many new methods which have emerged beyond the conventional filtering methods. Bag of words is one of the approach that obtained good result in classification of text. The challenges are diversified topics which can include technical as well as nontechnical topics. In case of technical topics words do not vary that frequently as compared to nontechnical topics like news. User's interest may vary from time to time or on daily basis so it is difficult to make a trend or pattern that user follows [6].

\section{Filtration of messages depending on policy}

In policy based approach there is more use of information retrieval method. The information is filtered mostly for textual documents and we data filtering. They also provide support for complex approaches involved in the online social network management. There is a policy framework which is till now provided to support administration and stipulation of policies which are expressed in place of limitations 
on the resource which can be interpreted by machine which is supplied by web based languages [6].

\section{Classification of machine learning with non-neutral} This is the method where classification is based on two level that is it is hierarchical approach. In the first level messages are categorizes into binary neutral or non-neutral. The second level categorization follows first level to perform finer classification. After the second level classification each message is assigned a category and each non-neutral message is assigned a class for further classification which is a step-by-step membership to the message [6].

\section{Radial Basis function networks (RBFN) using online setup}

The whole aim of this overall work is proposing a system which comes up with the firewall which automatically filters the messages and categorizes them automatically on the basis of available history and data and filters the undesirable messages from user walls. It explores a machine learning classification technique which automatically gives messages a category based on their data or content. The main efforts in structuring a short text classifier are focused on the set of in distinguished features [6].

\section{Delay of message in MANET}

In the mobile ad-hoc networks which are scanty the delay is accurately measured where nodes forward the messages. There are only two parameters for input which are the number of nodes and the second is the time over which two mobiles will come in each other's range for conversation purpose. The calculations for these are obtained using two protocols where one is two-hop multicopy and the other is unrestricted multicopy protocols. When the message is delivered. Even though the model is too simple it determines the delay accurately for the mobile nodes as well as the parameters which decides the time when two mobiles come into range for conversation with each other [6].

\section{Filtered Wall layer using the social Network Man- ager}

The social network manager layer aims to support fundamental functionalities using the online social network applications. It may also require the support of graphical user interface additionally. The neural model which isolates the messages of interest can be inserted into the two layers. Each category estimations are produced from RBFN by classifying the short messages [6].

principal directions given in this approach can satisfy the on-line detecting demand. A new arriving instance will be marked if its suspicious score is higher than the mean plus a specified multiple of the standard deviation. This online anomaly detection approach is well suited for online applications as it overcomes the computation issues and utilizes the memory efficiently [3].

\section{CONCLUSION}

Here, we studied different approaches for filtering the messages on online social network to provide the user security from unwanted messages and restricting the people from posting data which is not of interest on user wall. Primarily there us a use of neural and nonneural based technique for classification. Filtering rules and blacklist management are of the utmost important in the proposed architecture. It enhances the system performance effectively and precision as well as flexibility for classification of the messages is increased [5].

\section{REFERENCES}

[1] Peter W. Foltz and Susan T. Dumais, "Personalized Information Delivery: An Analysis of information filtering methods" December 1992/Vol.35, No.12.

[2] F. Sebastiani, "Machine Learning in Automated Text Categorization," ACM Computing Surveys, vol. 34, no. 1, pp. 1-47, 2002.

[3] P.J. Hayes, P.M. Andersen, I.B. Nirenburg, and L.M. Schmandt, "Tcs: A Shell for Content-Based Text Categorization," Proc. Sixth IEEE Conf. Artificial Intelligence Applications (CAIA '90), pp. 320-326, 1990.

[4] Foltz, P.W. Using Latent Semantic Indexing for information filtering. In Proceedings of the ACM Conference on Office Information Systems (Boston, Apr. 2527). ACM/SIGOIS, New York, 1990, pp. 40-47.

[5] Dhruv Vashistha,Sivagami.G. "Filtering Undesired Messages from Online Social Networks: A Content Based Filtering Approach" (IJCSIT) International Journal of Computer Science and Information Technologies, Vol. 5 (2) , 2014, 2046-2049.

[6] Kumutha G. "A System Can Filter the Unwanted Messages From OSN Walls" International Journal of scientific research and management (IJSRM) ||Volume||2||Issue||5 ||Pages|| 836-841 ||2014|| Website: www.ijsrm.in ISSN (e): 2321-3418. 